\title{
Media, Health Workers, and Policy Makers' Relationship and Their Impact on Antimalarial Policy Adoption: A Population Genetics Perspective
}

\author{
Allen Malisa, ${ }^{1,2}$ Richard Pearce, ${ }^{3}$ Benezeth Mutayoba, ${ }^{4}$ Salim Abdullah, ${ }^{2}$ Hassan Mshinda, ${ }^{2}$ \\ Patrick Kachur, ${ }^{5}$ Peter Bloland, ${ }^{5}$ and Cally Roper ${ }^{3}$ \\ ${ }^{1}$ Department of Biological Sciences, Faculty of Science, Sokoine University of Agriculture, P.O. Box 3038, Morogoro, Tanzania \\ ${ }^{2}$ Ifakara Health Institute (IHI), Research and Development Center (RDC), P.O. Box 53, Ifakara, Kilombero District, Tanzania \\ ${ }^{3}$ London School of Hygiene and Tropical Medicine, Pathogen Molecular Biology Unit, Department of Infectious Tropical Diseases, \\ Keppel Street, London WC1E 7HT, UK \\ ${ }^{4}$ Department of Veterinary Physiology, Biochemistry, Pharmacology and Toxicology, Faculty of Veterinary Medicine, \\ Sokoine University of Agriculture, P.O. Box 3017, Morogoro, Tanzania \\ ${ }^{5}$ Malaria Branch, Division of Parasitic Diseases, National Center for Infectious Diseases, \\ Centers for Disease Control and Prevention (CDC), Atlanta, GA 30341, USA
}

Correspondence should be addressed to Allen Malisa, malisa56@yahoo.com

Received 22 January 2011; Revised 19 April 2011; Accepted 26 April 2011

Academic Editor: Paul Wenzel Geissler

Copyright ( $) 2011$ Allen Malisa et al. This is an open access article distributed under the Creative Commons Attribution License, which permits unrestricted use, distribution, and reproduction in any medium, provided the original work is properly cited.

Drug resistance negatively impacts malaria treatments, making treatment policy revision unavoidable. So far, studies relating sociopolitical and technical issues on policy change with malaria parasite genetic change are lacking. We have quantified the effect of malaria treatment policy on drug pressure and the influence of the media, policy makers, and health worker relationship on parasite population genetic change in Kilombro/Ulanga district. Cross-sectional surveys of asymptomatic infections conducted before, during and after the switch from chloroquine to sulphadoxine/pyrimethamine were used for genetic analysis of SP resistance genes in 4,513 asymptomatic infections identified, and their frequency change was compared with retrospective study of the documented process of policy change. Highly significant changes of $d h f r$ and $d h p s$ resistance alleles occurred within one year of switch to SP first line, followed by a decline of their rate of selection caused by reduction of SP usage, as a result of negative media reports on SP usage and lack of adequate preparations.

\section{Introduction}

Malaria remains the world's most important tropical parasitic disease and one of the major public health challenges in the poorest countries of the world, particularly in sub-Saharan Africa, as the prospect of an effective vaccine remains uncertain. Although some African countries have increased their spending in health along with the support from the Global Fund to Fight AIDS, TB, and Malaria (GFATM) and other partners, development assistance has been routed largely through public channels, whereas affected individuals seek treatment mostly through the private sector [1]. Consequently, the rising cost of medical services and the increasing trends of drug resistance raise critical public health concerns, as this constrains the provision of adequate health care in countries where the disease is endemic [1]. Evidence on Plasmodium falciparum resistance to chloroquine (CQ) between 1980s and early 2000 prompted most countries where malaria is endemic to revise their treatment guidelines, most of them replacing it with sulfadoxine-pyrime (SP) [2, 3]. In Tanzania, CQ was officially replaced with SP in 2001, apparently as an interim policy [4]. However, SP resistance became widespread shortly after its adoption as first-line treatment, because its resistance had already been established in Tanzania and the neighbouring countries [5-8], and also, because it had been used as the second-line treatment for malaria in Tanzania until 2001, when it became the first-line treatment drug. Its 
long half-life could also have contributed to the observed rapid development of resistance [9]. Most malaria endemic countries replaced SP with artemisinin-based combination therapy (ACT) between the year 2002 and 2008. Tanzania replaced SP with artemether + lumefantrine (ALU) as the firstline national antimalarial treatment in 2006.

Treatment of malaria in Tanzania is typically guided by official recommendations from the Ministry of Health and Social Welfare (MOHSW) regarding drugs of choice for various situations. "First-line" treatment refers to the drug officially recommended as the drug of first choice for the treatment of uncomplicated malaria. "Second-line" treatment refers to the drug officially recommended as an alternative primarily to be used for treatment of patients in whom the first-line treatment failed to clear the infection and other select patients (such as those who are hypersensitive to the first-line treatment). "Third-line" treatment typically refers to the drug recommended for severely ill patients (a rescue drug). In practice, few treatment failures are recognized, and patients are often moved directly from first to third-line treatment, consequently, little second-line drug is used compared to the first-line drug. SP replaced CQ as the recommended first-line treatment on the Tanzanian mainland in August 2001, after 18 years of its use as a secondline treatment since 1983.

Policy change is both an expensive and difficult process, and many endemic countries are already faced with difficult decisions on how to replace ineffective antimalarial drugs in use with more efficacious, but also more costly alternatives. One of the greatest challenges in this decision-making process is the fear that resistance will develop rapidly to the replacement drug, initiating an endless cycle of drug replacement and escalating costs.

Studies conducted one year after adoption of SP as the first-line antimalarial treatment policy in Tanzania indicate that inadequate evidence-based education and sensitization of health workers together with negative media reports negatively impacted the adoption of the new policy $[4,10$, $11]$.

Theory predicts that we can protect the efficacy of future antimalarials by changing treatment practice or drug formulation, but the potential success of such interventions rests upon their impact on drug pressure in the field [12]. Mathematical models [13] predict that drug coverage/extensive drug usage is the primary determinant of drug pressure, and the driving force behind the evolution of drug resistance [14, 15]. Research findings have established that resistance to $C Q$ and SP results from accumulation of multiple mutations in the respective target gene(s). Once formed, these mutations spread within and between continents $[8,16-18]$.

We have systematically followed the rate of selection of SP resistance alleles in a one-year prior and five years after adoption of SP as the first-line antimalarial treatment in Tanzania. From the molecular genetics perspective, we show that the trend displayed by resistant alleles together with linkage disequilibrium between double $d h p s$ and triple $d h f r$ resistant alleles correlates with published reports of media $[4,10,11]$ and health worker's influence on policy adoption [4].

\section{Materials and Methods}

2.1. Study Area, Subjects, and Samples. Community surveys were conducted during July, August, and September of 2000, 2001, 2002, 2004, 2005, and 2006 in Kilombero (population. $=220,000)$ and Ulanga (population $=160,000$ ). The surveys were part of a large combination therapy pilot implementation programme in Tanzania, the Interdisciplinary Monitoring Programme for Antimalarial Combination Therapy (IMPACT-TZ). For the purpose of the study, Kilombero and Ulanga districts were treated as a single district, because population movement between these two districts is high and the study population spans the border region. Plasmodium falciparum malaria transmission in the study area is intense (with an estimated entomological inoculation rate of 367 infectious bites per person per year [19] and perennial with some seasonal fluctuation). A total of 24,212 adults and children belonging to randomly selected households participated in the study. A finger-prick blood sample for blood slide and filter paper bloodspot were collected from each individual in the household. The filter paper bloodspots were air-dried and put in self-sealing plastic bags with desiccant and stored on dry area at room temperature. All blood slide samples were screened by microscopy for P. falciparum. Bloodspots from microscopically positive subjects were selected and preserved at room temperature for molecular genotyping.

2.2. Antimalarial Drug Policy. Prior to the first national antimalarial policy change in Tanzania in August 2001, the first-line treatment drug was CQ, SP was the secondline, while quinine $(\mathrm{Q})$ was the third-line treatment. The policy change replaced CQ with SP as first-line treatment and SP was replaced with amodiaquine (AQ) as secondline treatment, while $\mathrm{Q}$ continued serving as the thirdline treatment. Issues surrounding the 2001 policy change are detailed in [4]. The policy decision was supported by research evidence indicating an intolerable parasite resistance to CQ and clinical CQ treatment failure rates of above $52 \%$, as compared to SP and AQ failure rate of about $9.5 \%$ and less than $4.6 \%$, respectively. Research also indicated that since SP was also facing rising resistance trend, the need for a more effective drug was indispensable, but for an interim 5-10-year period, it was justifiable to recommend SP that was relatively more cost effective than CQ and AQ. The government launched the policy change considering that studies (ethically approved by the Ministry of Health) on therapeutic efficacy and cost effectiveness of artemisinin drug combination therapies were underway. Nevertheless, the process of communicating research results and recommendations to policy-making authorities involved critical debates between policy makers and researchers, among the researchers themselves and between the researchers and general practitioners, the speculative media reports on SP side effects and reservations by the general public concerning the rationale for policy change, when to change, and to which drug of choice.

The second national antimalarial policy change was implemented in 2006, whereby artemether and lumefantrine (ALU) replaced SP as the first-line treatment. However, the 
policy remained silent about the second-line and the thirdline treatments although in practice, $\mathrm{Q}$ is used as both the second-line and rescue drug, because when a patient on ALU therapy fails to clear parasite, he/she is moved straight to Q.

Overall, while the dynamics surrounding the government's policy to replace CQ with SP in 2001 have been well documented [4], there is very little published data on the process of change from SP to ALu. The evidence supporting the change came from a number of in vitro and in vivo studies showing unacceptable levels of parasite resistance to SP and the IMPACT-Tanzania studies which piloted the use of ACT in rural Tanzania [20].

2.3. Ethical Consideration. Scientific and ethical clearance was granted from the Medical Research Council of the National Institute for Medical Research in Tanzania, the Centers for Disease Control and Prevention, USA, and the London School of Hygiene and Tropical Medicine. Consent was obtained from all individuals or their guardians before collection of samples.

2.4. DNA Extraction. The DNA was extracted from bloodspots dried on filter papers. A section of the dried blood spot filter paper was excised using a sterile blade or scissors and soaked in a $1 \mathrm{~mL}$ of $0.5 \%$ saponin in $1 \mathrm{x}$ phosphate buffered saline (PBS) overnight in a 96-deepwell plate. The segment was then washed twice in $1 \mathrm{~mL}$ of $1 \mathrm{x}$ PBS and was finally boiled for $8 \mathrm{~min}$ in $100 \mu \mathrm{L}$ PCR quality water with $50 \mu \mathrm{L}$ of $20 \%$ chelex suspension ( $\mathrm{pH}$ 9.5).

2.5. PCR Amplification. Nested PCR was used to amplify a 594 base pair (bp) fragment of $d h f r$ and a $711 \mathrm{bp}$ fragment of dhps each containing the sequence, where mutations are found. Primer sequences and PCR reaction conditions were previously described in [7]. PCR was performed in 96 well plates with $25-\mu \mathrm{L}$ PCR reaction volumes containing final concentrations of $0.25 \mu \mathrm{M}$ oligonucleotide primers, $2 \mathrm{mM} \mathrm{MgCl} 2,250 \mu \mathrm{M}$ each deoxyribonucleotide triphosphate (dNTPs), and $1 \mathrm{x}$ Taq polymerase. $1 \mu \mathrm{L}$ of DNA template was used in the outer (primary) PCR reaction mixture for $d h f r$ and $d h p s$ amplifications. For the inner (secondary) $d h p s$ reactions $1 \mu \mathrm{L}$ of the outer PCR product was used. The outer $d h f r$ PCR products were diluted three fold before a $1 \mu \mathrm{L}$ was introduced into the inner PCR reaction mixtures.

2.6. Molecular Genotyping of Point Mutations by Sequence Specific Oligonucleotide Probing (SSOP). The amplified PCR products were screened for $d h f r$ and $d h p s$ sequence variants at 10 loci, where single nucleotide polymorphisms (SNPs) are known. The sequence changes (and the amino acid substitutions they code for) are summarised in Table 1.

PCR products were spotted in a 12 by 8 -grid and cross linked onto nylon membranes and probed for sequence polymorphisms by hybridisation to specific oligonucleotide probes described previously [7]. For analysis of samples collected in 2000, the visualization of hybridised digoxygenin labelled probes on membranes was performed by the alkaline phosphatase-catalysed breakdown of the CSPD substrate
TABLE 1: The nucleotide and amino acid substitutions at (a) dhfr and (b) dhps genes screened for by PCR-SSOP.

$$
\text { (a) } d h f r
$$

\begin{tabular}{lccccc}
\hline Codon & 50 & 51 & 59 & 108 & 164 \\
\hline \multirow{4}{*}{ Wild type } & Cys (C) & Asn (N) & Cys (C) & Ser (S) & Ile (I) \\
& TGT & AAT & TGT & AGC & ATA \\
& & AAC & & & \\
& Arg (R) & Ile (I) & Arg (R) & Asn (N) & Leu (L) \\
Mutant & CGT & ATT & CGT & AAC & TTA \\
& & & & Thr (T) & \\
& & & & ACC & \\
\hline
\end{tabular}

(b) dhps

\begin{tabular}{lccccc}
\hline Codon & 436 & 437 & 540 & 581 & 613 \\
\hline \multirow{2}{*}{ Wild type } & Ser (S) & Ala (A) & Lys (K) & Ala (A) & Ala (A) \\
& TCT & GCT & AAA & GCG & GCC \\
& Phe (F) & Gly (G) & Glu (E) & Gly (G) & Ser (S) \\
& TTT & GGT & GAA & GGG & TCC \\
Mutant & Ala (A) & & & & Thr (T) \\
& GCT & & & & ACC \\
& Cys (C) & & & & \\
\hline
\end{tabular}

(Roche Boehringer Mannheim, Mannheim, Germany) and visualised by exposure on Hyperfilm-ECL (Amersham Pharmacia Biotech, Little Chalfont, Buckinghamshire, United Kingdom), according to Boehringer Mannheim recommendations and previously described in [21]. For analysis of samples collected in 2001 and 2002, the probed blots were visualised using ECF substrate and detection using a phosphoimager (STORM). Inspection of autoradiographic films was carried out by light box illumination, while the phosphoimager output was recorded through viewing of digitally captured images of chemiflourescent signal. The change in the method by which probe hybridisation signal was visualised did not affect the results in any way since the probes and hybridisation conditions were unchanged.

The stringency and specificity of the hybridisation process was confirmed by inspection of a series of 4 controls with a known single genotype variant sequence. All blots with nonspecifically bound probes were stripped and reprobed. A SNP was considered to be present in the PCR product when the intensity of signal was higher than that of the background. The blots were scored independently by two people.

In our analysis, we aimed to establish the relative abundance of different point mutation haplotypes at $d h f r$ and dhps. Since bloodstage P. falciparum is haploid, this is very straightforward when an infection consists of a single genotype, because only one form of sequence at every SNP locus is seen. When infections are composed of multiple genotypes a mixture of different sequence variants occur making the inference of point mutation haplotypes within that infection more difficult.

The presence, absence, and relative abundance of hybridisation signals for every probe were recorded at each 
TABLE 2: Annual survey population, proportions that were malaria positive and their PCR amplification outcome for the period between 2000-2006 in Kilombero/Ulanga population.

\begin{tabular}{|c|c|c|c|c|c|c|}
\hline & 2000 & 2001 & 2002 & 2004 & 2005 & 2006 \\
\hline Survey population & 3,289 & 3,197 & 4,098 & 4,122 & 5,006 & 4,500 \\
\hline P. falciparum positive & 955 & 580 & 875 & 972 & 486 & 645 \\
\hline PCR amplified $d h f r$ & 404 & 488 & 686 & 660 & 374 & 294 \\
\hline PCR amplified $d h p s$ & 444 & 347 & 720 & 712 & 407 & 323 \\
\hline Single or majority $d h f r$ & 376 & 238 & 489 & 540 & 267 & 275 \\
\hline Single or majority $d h p s$ & 365 & 294 & 603 & 562 & 293 & 275 \\
\hline Single or majority $d h f r+d h p s$ & 190 & 138 & 381 & 381 & 283 & 206 \\
\hline
\end{tabular}

locus. A sample was considered to have a single haplotype when only one sequence variant was found at each locus. Blood samples were categorised as having a single, a majority or a mixture of sequence at every SNP locus. Majority and mixed genotype infections were differentiated according to the relative intensity of signal. To determine the relative abundance of different point mutation haplotypes in the parasite population, only one haplotype was counted from each infection and those mixed infections, where haplotypes could not be resolved were omitted from the calculation of haplotype frequencies. Hence, frequency data is based upon a subset of isolates which were unmixed or had a predominating majority haplotype. A breakdown of the proportions of isolates which were successfully PCR amplified and genotyped as single, majority or mixed haplotype infections is given in Table 2 .

2.7. Statistical Analysis. Statistical comparison of allele frequencies at $d h f r$ and $d h p s$ in the various sites was carried out using chi-squared analysis in STATA version 9.2. The calculation of binomial exact 95\% confidence intervals was carried out using STATA version 9.2. Linkage disequilibrium analysis was performed using Arlequin software.

\section{Results}

24,212 people were sampled and 4,513 asymptomatic infections identified. DNA was extracted from the 4,513 P. falciparum positive bloodspots and PCR of $d h f r$ and dhps performed once, giving a combined rate of PCR amplification success of $69 \%$ for both genes (Table 2 ). The amplified products were screened for all the variant sequences described in Table 1. Out of the 2,906 isolates which amplified for $d h f r$, $2,185(75 \%)$ were single or majority genotype infections and the point mutation haplotypes could easily be resolved. Of the 2,953 samples which amplified for dhps, 2,392 (81\%) were single or majority genotype with resolvable haplotypes.

3.1. Allelic Haplotypes at dhfr and dhps Genes. The point mutations found in the $d h f r$ gene were N51I, C59R, and $\mathrm{S} 108 \mathrm{~N}$ as reported elsewhere [12]. They were found in the following haplotypic arrangements CNCSI, CNCNI, CNRNI, CICNI, and CIRNI which are reported to be common throughout Eastern Africa, Tanzania [7], Malawi [22], Kenya [5, 23] and Uganda [24, 25].

Five mutations were found at dhps; S436A, S436F, S436C, $\mathrm{A} 437 \mathrm{G}$, and $\mathrm{K} 540 \mathrm{E}$ as reported elsewhere [12]. These were found in nine distinct haplotypic arrangements; five of which (SAKAA, AAKAA, SGEAA, SGKAA, and SAEAA) were described previously in isolates from East Africa [5, 7, 2325] while the remaining four (CAKAA, FAKAA, AAEAA, and FAEAA) were found in extremely low frequency and have not been reported before, presumably because of their rarity.

3.2. Haplotypes Conferring High Levels of Resistance. The $d h f r$ CIRNI haplotype and the dhps SGEAA haplotype have the greatest significance for SP efficacy; their frequency in the six successive surveys displayed three distinct phases as shown in Figures 1 and 2. Changes occurring under the CQ policy (phase 1: 2000 to 2001) are markedly different to those occurring under the SP policy (phase 2: 2001-2002). Likewise, the frequency of the dhps double mutant A437G K540E (shown in Figure 2) did not change significantly in phase 1, between 2000 and 2001 ( $P \geq$.497). Contrastingly, there was a highly significant change in phase 2 , between 2001 and 2002 ( $P \leq .0001)(95 \% \mathrm{CI})$. In phase 3, between 2002 and 2006 both the dhfr triple and the dhps double mutant alleles showed a marked retarded rate of selection of the two unlinked resistant alleles.

In a further subset of samples, where $d h f r$ and $d h p s$ haplotypes were both conclusively resolved, it was possible to measure the frequency of two locus genotypes.

In Figure 3, the frequency of the triple $d h f r+$ double $d h p s$ genotype for the six successive surveys is presented. As for the case of the $d h f r$ triple and the $d h p s$ double mutant alleles, the triple $d h f r+$ double $d h p s$ genotype showed three distinct phases. The frequency was around 0.05 in phase one and there was no change between 2000 and $2001(P \geq .824)$ but a remarkable 5 fold increase to frequency of 0.28 occurred in phase two between 2001 and $2002(P \leq .0001)$. In phase three, this two locus genotype (the triple $d h f r+$ double dhps) was characterised by a marked reduction of the rate of selection between 2002 to 2006 .

3.3. Haplotypes Conferring Intermediate Levels of Resistance. As well as the highly resistant dhps A437G K540E double mutant, a number of single 436 mutant alleles were recorded. 


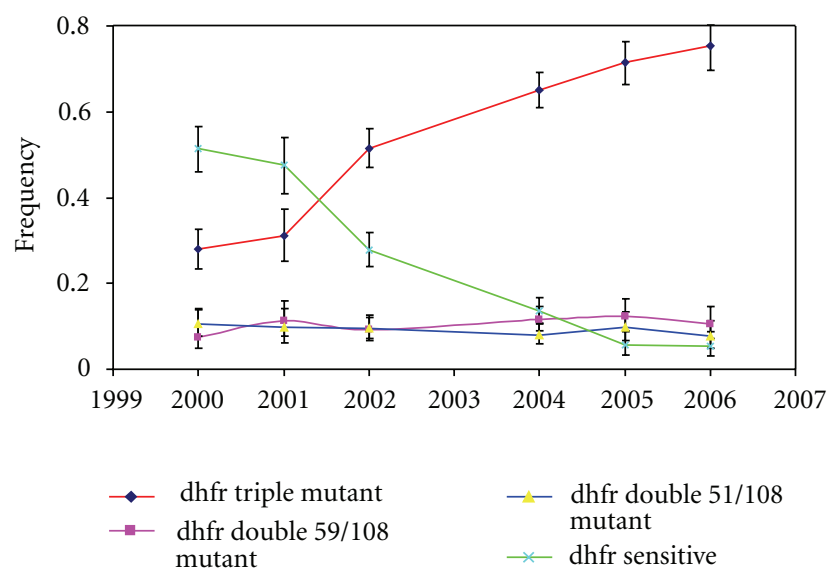

Figure 1: Changes in the frequency of pyrimethamine resistance gene $(d h f r)$ in Kilombero/Ulanga in six successive surveys (20002006).

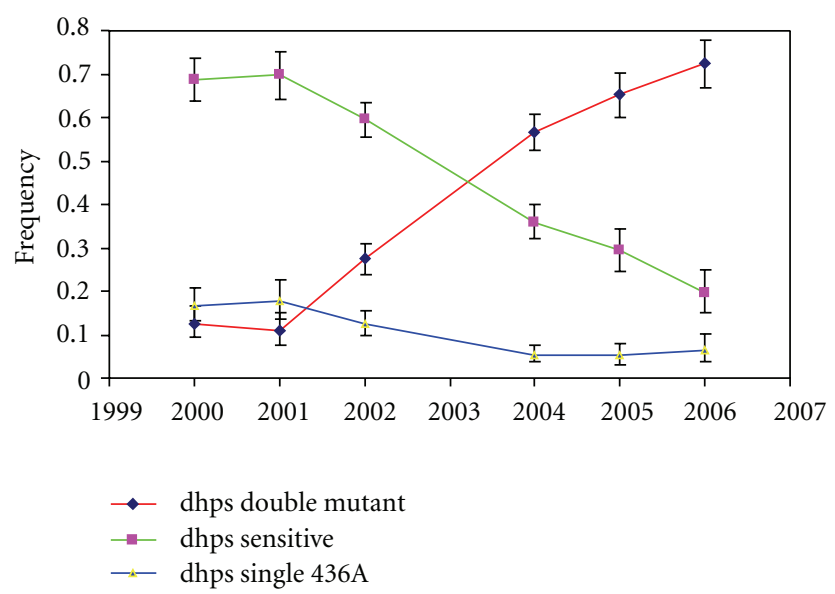

FIGURE 2: Changes in the frequency of Sulfadoxine resistance gene (dhps) in Kilombero/Ulanga between 2000-2006.

Among these, by far the most common was the S436A, which was consistently found at frequencies of $10 \%-20 \%$ in all six successive surveys. Figure 2 shows the frequencies of the sensitive S436A single mutant and the A437G K540E double mutant alleles through time. From these data, it is clear that the increased frequency of the double mutant allele displaced the sensitive allele, which decreased nonsignificantly in 2000$2001(P \geq .791)$ and significantly in 2001-2002 $(P \geq .003)$. Interestingly, the frequency of the A436A allele remained static in all time points in both districts.

The effect of drug policy changes on the frequency of sensitive and double mutant $d h f r$ alleles is shown in Figure 1. The increase of the triple mutant allele acted to displace sensitive alleles which showed a substantial but non significant decline in phase one (2000-2001) and phase three (2002-2006) $(P \geq .352)$ and a highly significant decline in phase two (2001-2002) ( $P \leq .0001)$. The double mutant $d h f r$ alleles, which confer intermediate levels of resistance in vitro [26], neither increased nor decreased, remaining at a

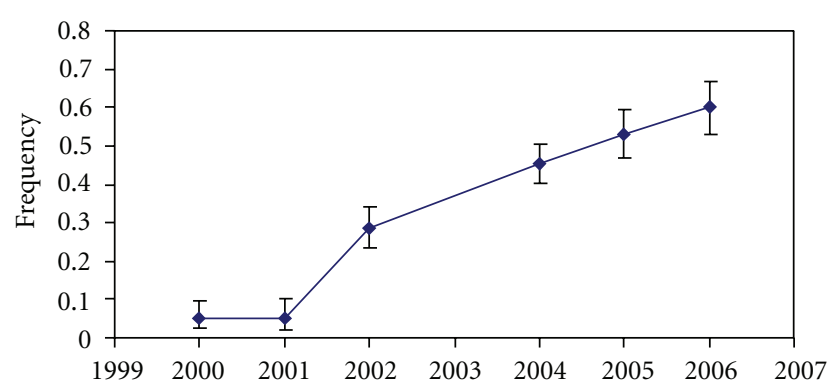

Figure 3: Changes in the frequency of the two locus genotype combination, $d h f r$ triple $+d h p s$ double mutant allele in Kilombero/Ulanga in six successive surveys (2000-2006).

TABle 3: The trend of the D' values (an index of linkage disequilibrium) and their $P$ values in the period between 2000 and 2006 in Kilombero/Ulanga population.

\begin{tabular}{lcc}
\hline \multirow{2}{*}{ Year of survey } & \multicolumn{2}{c}{ Kilombero/Ulanga } \\
& $D^{\prime}$ & $P$ \\
\hline 2000 & -0.5333 & .3307 \\
2001 & 0.0417 & .7539 \\
2002 & 0.3633 & $.00001^{* * * *}$ \\
2004 & 0.2948 & $.0001^{* * *}$ \\
2005 & 0.2595 & $.0001^{* * *}$ \\
2006 & 0.0814 & .2686 \\
\hline
\end{tabular}

* Indicates degree of significance.

frequency of around $10 \%$ in both districts throughout all surveys.

3.4. Linkage Disequilibrium (LD). To examine the effect of simultaneous selection by pyrimethamine on $d h f r$ and sulphadoxine on $d h p s$, we looked at two-locus genotypes sampled from Kilombero/Ulanga in the six successive surveys. Taking the subset of samples for which point mutation haplotypes could be unequivocally resolved for both genes, we compared the observed with expected frequencies generated from contingency tables. No significant linkage disequilibrium was found between $d h f r$ and $d h p s$ loci in either $2000\left(D^{\prime}=-0.5333, P \geq .3307\right)$ or $2001\left(D^{\prime}=\right.$ 0.0417, $P \geq$.75339) indicating that the drug selection pressure was insufficient to overcome the force of recombination. However, a highly significant LD $\left(D^{\prime}=0.3633\right.$, $P \leq .00001)$ was observed in 2002 indicating that the drug selection pressure was already sufficiently higher to overcome the effect of recombination. The degree of significance of the LD started to show a decline in $2004\left(D^{\prime}=0.2948\right.$, $P \leq .00001)$ and $2005\left(D^{\prime}=0.2595, P \leq .00001\right)$, and by 2006, there was no more significant $\operatorname{LD}\left(D^{\prime}=0.0814\right.$, $P \geq$.2686) (Table 3). This indicates that there was a gradual decline in drug pressure between 2002 and 2006.

\section{Discussion}

This study has generated results of six-year followup of $d h f r$ and dhps genetic changes in the P. falciparum in two 
rural districts of south eastern Tanzania. A high throughput sequence specific olignucleotide method was used to genotype point mutations present at the SP resistance genes, the $d h f r$ and $d h p s$ in population samples of $P$. falciparum infections collected annually from year 2000 to 2006 in Kilombero/Ulanga. Significant increase of the frequencies of resistant $d h f r$ and dhps haplotypes was observed after the change of the National antimalarial guidelines from firstline CQ to first-line SP. Clearly, changes of drug use policies implemented during the conduct of this study had impact on the genetic determinants of SP resistance.

4.1. Policy and Genetic Change. The study has demonstrated that the influence of the national antimalarial treatment policy change on the genetic composition of the malaria parasite population was profound. Dhfr and dhps allele frequency did not change significantly during 12 months (year 2000 to 2001) of CQ as first-line and SP as secondline, but after the switch to SP as first-line treatment (year 2001 to 2002), the frequency of the triple mutant $d h f r$ allele increased from $31 \%-52 \%$, while the frequency of the double mutant dhps allele increased 3-fold. A combination of these alleles is predictive of in vivo failure of SP treatment $[5,6,27]$, and the rapid increase of this genotype from $5 \%-29 \%$ between 2001-2002 suggests that the outlook for SP efficacy in this region was deteriorating. In fact, this observation was borne out by the results of SP treatment efficacy monitoring in south-eastern Tanzania during 2003 which found that $49 \%$ of SP treatments failed by day 28 [28]. Due to this high rate of SP resistance, national policy decision was switched to the recommended firstline treatment to artemether and lumefantrine in 2006. The observed escalation of SP resistance alleles is attributed to the policy change which brought about immediate shift in treatment practice, characterised by increased demand of SP both in the public and private sector leading to intense SP drug pressure [12]. Again, because the change of policy was nation wide, the same SP pressure was likely applying to the districts surrounding the study area, narrowing the likelihood of significant higher selection and hence flow of resistant parasites from the neighbouring districts to the study area.

4.2. Correlation between the Trend of SP Usage and the Levels of dhfr and dhps Resistance Alleles. Prior to the introduction of SP as first-line therapy (between 2000 and 2001), SP was widely available for self treatment via purchase from pharmacies, shops [29] as well as through formal health facilities as the second-line treatment. The SP drug pressure exerted during this period was only sufficient to maintain $d h f r$ and $d h p s$ resistance alleles at constant levels. By contrast, following the introduction of SP as first-line in 2001, there was dramatic increase in SP use [29] leading to intense SP pressure and escalation of $d h f r$ and $d h p s$ resistance alleles. Although SP is currently being replaced as a first-line drug for treatment of malaria in Tanzania and much of the rest of Africa, it will continue to be used in intermittent preventive treatment of infants and pregnant women [30]. The drug pressure applied when SP is used solely in intermittent programmes of treatment in infants and pregnant women will probably more closely resemble the situation observed between 2000-2001, when SP was second-line treatment than during the period it was used as the first-line treatment. The consequences of more restricted use for future efficacy of SP are therefore two fold. Firstly, rates of resistance allele frequency change are likely to stabilise as a result of reduction of SP selection pressure. Secondly, the reduction of selection is predicted to disassociate the triple mutant $d h f r$ allele and double mutant $d h p s$ allele which will reduce the frequency of highly pyrimethamine and sulphadoxine resistant parasites in the P. falciparum population at large.

4.3. Linkage Disequilibrium. Direct observation such as plasma drug levels in the patient gives the best direct measure of drug selection in the population and it would be interesting to examine this in comparison to the LD measures. LD on the other hand is known to give a good indirect indication of SP selection pressure, and hence, the lack of significant LD in Kilombero/Ulanga population in 2000 and 2001 when CQ was the first-line treatment conforms to expectations. In 2002, however, the significant LD emerged indicating intensification of drug pressure following country wide distribution and use of the SP as the new first-line treatment [29]. Contrary to our expectations, just one year of SP use as first-line treatment, a noticeable decline in the rate of increase of resistant $d h f r$ and $d h p s$ alleles along with decreasing significance of the LD was observed and continued in the rest of surveys. By 2006 the LD was no longer significant indicating that the SP drug selection strength was insufficient to keep the two alleles, the triple $d h f r$ and double $d h p s$ alleles in combination. To understand the reason for the three phase pattern of progression of SP resistance alleles observed in this study, we retrospectively reviewed the process of policy change in Tanzania in 2001, from CQ to SP first-line treatment.

4.4. Changes in Government Guidelines and the Public Response. In order to explain the underlying cause of reduction of SP selection strength just one year after SP first-line adoption in Tanzania, we retrospectively reviewed the process of policy change from CQ to SP first line. The study also examined retrospectively the public perception and the role of media in informing the public, who are the end point consumers of the policy. Accordingly, The National Task Force on Antimalarial Drug Policy was formulated in May 1999 as a subcommittee of the National Malaria Advisory Committee (NMAC) in consultation with the East African Network for Monitoring Antimalarial Treatments (EANMAT) and WHO country office in Dar es Salaam [4].

On 23 July 1999, the task force developed a three-page summary, drawing on evidence from clinical trials in the sentinel sites. It was recommended that the decision to change the policy should be interim because of increasing evidence of high SP resistance in various parts of the country such as Muheza and Kilombero districts [31]. After the presentation of the new policy by the task force, a series of news papers and some private radio stations began to inform the public that CQ was no longer a recommended 
drug for malaria treatment and that the government was considering replacing it with a new drug. This information caused public concern and debates erupted in different parts of the country about the rationality for the change $[4,10,11]$. Those involved were the general public, the research community, traders, the pharmaceutical industry, and health-care providers in both the public and private health facilities.

In an attempt to maintain public confidence, the Government through the Ministry of Health gave out a press release that indicated its stand concerning the treatment guidelines to be followed while strategies were underway to make an appropriate decision. Anecdotal evidence indicates that many health professionals were unaware of the extent of resistance to CQ and did not perceive an urgent need for change [3].

While WHO recommends change to an alternative drug when the treatment failure rates with the first-line drug reaches $25 \%$, evidence from different sentinel sites in the country indicated that up to the time of policy change, CQ treatment failure rate had already reached $52 \%$ (ranging $28 \%-72 \%$ ), 9.5\% for SP (ranging 6\%-32\%), while treatment failure to other drugs such as $\mathrm{AQ}$ and $\mathrm{Q}$ was less than $4.6 \%$ (ranging from 3.5\%-6\%) [32]. More criticism ensued between the Ministry of health, the local newspapers and the research community on the effectiveness of CQ. While the public were advised to remain patient until the government gathered sufficient evidence, researchers continued to disseminate information indicating high levels of resistance and suggesting for replacement of CQ with a more suitable drug [4]. The government's official announcement of the policy change came out of the media in 2000 although the actual implementation officially started on 1st August 2001.

Clearly, there were big differences between different actors which spurred a great deal of challenges in the long term uptake and support of the new policy down to the communities. A study conducted in 2002 (one year of policy adoption) reported widespread fear and negative perceptions about the use of SP [11]. Lack of educational campaign from the Ministry of Health and domination of the media with reports of adverse SP reaction, led to a growing trend of lack of confidence in SP use as demonstrated by the public $[10,11]$. The trend of events in the policy adoption and implementation described here corroborates well with the genetic events described in Figures 1 and 2. Within the first year of policy implementation, usage of SP was highest (intense selection pressure with significant positive LD in the population). However, as adverse reaction to SP continued to mount, and were exaggerated by the media, the drug gradually lost popularity. It is also very possible that as SP resistance intensified over time, an increasing number of SP users reverted to alternative antimalarials again reducing SP selection pressure.

\section{Conclusions and Recommendations}

The national policy change brought about an immediate shift in treatment practice and this in turn had a highly significant impact on drug pressure. This shows that even in rural areas where access to treatment is imperfect and treatment coverage relatively low, first-line SP selection is sufficiently strong to rapidly change the genetic composition of the parasite population in one year. Furthermore, selection applied was strong enough to overcome recombination pressure and create linkage disequilibrium between the unlinked genetic determinants of resistance to the two co-administered drugs (sulfadoxine and pyrimethamine), showing that recombination is not a barrier to the evolution of multidrug resistance in high endemicity settings.

The data presented here show that both the media and health workers play a key role to modify the political technical and social values of policy adoption success. The need to consider when and how research information should be communicated between different stake holders is very crucial and should enable avoiding similar dilemmas in future.

\section{Role of the Funding Source}

This study was funded through an interagency agreement between the United States Agency for International Development (USAID) and CDC and a cooperative agreement between CDC and the Ifakara Health Research and Development Center (IHRDC). USAID did not participate in the design, collection, analysis, or interpretation of the data, in the writing of the paper, or in the decision to submit for publication.

\section{Acknowledgments}

The authors would like to thank all of the people who participated in the cross-sectional surveys in Kilombero and Ulanga districts in Tanzania. The Interdisciplinary Monitoring Project for Antimalarial Combination Therapy in Tanzania (IMPACT-Tz) was funded by USAID, CDC, and Wellcome Trust, and the coprincipal investators were P. Bloland and S. Abdulla. C. Roper and R. Pearce were supported by a Wellcome Trust Fellowship (no. 060714) awarded to C. Loper.

\section{References}

[1] O. Adeyi and R. Atun, "Universal access to malaria medicines: innovation in financing and delivery," The Lancet, vol. 376, no. 9755, pp. 1869-1871, 2010.

[2] P. B. Bloland, E. M. Lackritz, P. N. Kazembe, J. B. O. Were, R. Steketee, and C. C. Campbell, "Beyond chloroquine: implications of drug resistance for evaluating malaria therapy efficacy and treatment policy in Africa," Journal of Infectious Diseases, vol. 167, no. 4, pp. 932-937, 1993.

[3] P. B. Bloland, P. N. Kazembe, A. J. Oloo, B. Himonga, L. M. Barat, and T. K. Ruebush, "Chloroquine in Africa: critical assessment and recommendations for monitoring and evaluating chloroquine therapy efficacy in sub-Saharan Africa," Tropical Medicine and International Health, vol. 3, no. 7, pp. 543-552, 1998.

[4] G. M. Mubyazi and M. A. Gonzalez-Block, "Research influence on antimalarial drug policy change in Tanzania: case study of replacing chloroquine with sulfadoxinepyrimethamine as the first-line drug," Malaria Journal, vol. 4, pp. 51-65, 2005. 
[5] S. A. Omar, I. S. Adagu, and D. C. Warhurst, "Can pretreatment screening for $d h p s$ and $d h f r$ point mutations in Plasmodium falciparum infections be used to predict sulfadoxinepyrimethamine treatment failure?" Transactions of the Royal Society of Tropical Medicine and Hygiene, vol. 95, no. 3, pp. 315-319, 2001.

[6] J. G. Kublin, F. K. Dzinjalamala, D. D. Kamwendo et al., "Molecular markers for treatment failure of sulfadoxinepyrimethamine and chlorproguanil-dapsone for falciparum malaria and a model for practical application in Africa," Journal of Infectious Diseases, vol. 85, no. 3, pp. 380-388, 2002.

[7] R. J. Pearce, C. Drakeley, D. Chandramohan, F. Mosha, and C. Roper, "Molecular determination of point mutation haplotypes in the dihydrofolate reductase and dihydropteroate synthase of Plasmodium falciparum in three districts of Northern Tanzania," Antimicrobial Agents and Chemotherapy, vol. 47, no. 4, pp. 1347-1354, 2003.

[8] C. Roper, R. Pearce, B. Bredenkamp et al., "Antifolate antimalarial resistance in southeast Africa: a population-based analysis," Lancet, vol. 361, no. 9364, pp. 1174-1181, 2003.

[9] A. M. Nzila, E. Nduati, E. K. Mberu et al., "Molecular evidence of greater selective pressure for drug resistance exerted by the long-acting antifolate pyrimethamine/sulfadoxine compared with the shorter-acting chlorproguanil/dapsone on Kenyan Plasmodium falciparum," Journal of Infectious Diseases, vol. 181, no. 6, pp. 2023-2028, 2000.

[10] J. Eriksen, S. E. D. Nsimba, O. M. S. Minzi et al., "Adoption of the new antimalarial drug policy in Tanzania-a crosssectional study in the community," Tropical Medicine and International Health, vol. 10, no. 10, pp. 1038-1046, 2005.

[11] S. E. D. Nsimba, "How sulfadoxine-pyrimethamine (SP) was perceived in some rural communities after phasing out chloroquine $(\mathrm{CQ})$ as a first-line drug for uncomplicated malaria in Tanzania: lessons to learn towards moving from monotherapy to fixed combination therapy," Journal of Ethnobiology and Ethnomedicine, vol. 2, pp. 5-13, 2006.

[12] A. L. Malisa, R. J. Pearce, S. Abdulla et al., "Drug coverage in treatment of malaria and the consequences for resistance evolution-evidence from the use of sulphadoxine/pyrimethamine," Malaria Journal, vol. 9, no. 1, p. 190, 2010.

[13] I. M. Hastings, "A model for the origins and spread of drugresistant malaria," Parasitology, vol. 115, no. 2, pp. 133-141, 1997.

[14] N. J. White, “Antimalarial drug resistance," Journal of Clinical Investigation, vol. 113, no. 8, pp. 1084-1092, 2004.

[15] N. Noranate, R. Durand, A. Tall et al., "Rapid dissemination of Plasmodium falciparum drug resistance despite strictly controlled antimalarial use," PLOS ONE, vol. 2, no. 1, Article ID e139, 2007.

[16] J. C. Wootton, X. Feng, M. T. Ferdig et al., "Genetic diversity and chloroquine selective sweeps in Plasmodium falciparum," Nature, vol. 418, no. 6895, pp. 320-323, 2002.

[17] C. Roper, R. Pearce, S. Nair, B. Sharp, F. Nosten, and T. Anderson, "Intercontinental spread of pyrimethamineresistant malaria," Science, vol. 305, no. 5687, p. 1124, 2004.

[18] R. Pearce, H. Pota, M. B. Evehe et al., "Dispersal of drug resistant dhps reveals regional migration patterns among African P. falciparum," Plos Biology, vol. 6, no. 4, Article ID e1000055, 2009.

[19] S. I. Hay, D. J. Rogers, J. F. Toomer, and R. W. Snow, "Annual Plasmodium falciparum entomological inoculation rates (EIR) across Africa: literature survey, internet access and review," Transactions of the Royal Society of Tropical Medicine and Hygiene, vol. 94, no. 2, pp. 113-126, 2001.

[20] S. P. Kachur, R. A. Khatib, E. Kaizer, S. S. Fox, S. M. Abdulla, and P. B. Bloland, "Adherence to antimalarial combination therapy with sulfadoxine/pyrimethamine and artesunate in rural Tanzania," American Journal of Tropical Medicine and Hygiene, vol. 71, no. 6, pp. 715-722, 2004.

[21] D. J. Conway, C. Roper, A. M. J. Oduola et al., "High recombination rate in natural populations of Plasmodium falciparum," Proceedings of the National Academy of Sciences of the United States of America, vol. 96, no. 8, pp. 4506-4511, 1999.

[22] B. Bwijo, A. Kaneko, M. Takechi et al., "High prevalence of quintuple mutant dhps/dhfr genes in Plasmodium falciparum infections seven years after introduction of sulfadoxine and pyrimethamine as first line treatment in Malawi," Acta Tropica, vol. 85, no. 3, pp. 363-373, 2003.

[23] A. M. Nzila, E. K. Mberu, J. Sulo et al., "Towards an understanding of the mechanism of pyrimethamine-sulfadoxine resistance in Plasmodium falciparum: genotyping of dihydrofolate reductase and dihydropteroate synthase of Kenyan parasites," Antimicrobial Agents and Chemotherapy, vol. 44, no. 4, pp. 991-996, 2000.

[24] D. Kyabayinze, A. Cattamanchi, M. R. Kamya, P. J. Rosenthal, and G. Dorsey, "Validation of a simplified method for using molecular markers to predict sulfadoxine-pyrimethamine treatment failure in African children with falciparum malaria," The American Journal of Tropical Medicine and Hygiene, vol. 69, no. 3, pp. 247-252, 2003.

[25] H. Sendagire, D. Kyabayinze, G. Swedberg, and F. Kironde, "Plasmodium falciparum: higher incidence of molecular resistance markers for sulphadoxine than for pyrimethamine in Kasangati, Uganda," Tropical Medicine and International Health, vol. 10, no. 6, pp. 537-543, 2005.

[26] D. Menard, F. Yapou, A. Manirakiza, D. Djalle, M. D. Matsika-Claquin, and A. Talarmin, "Polymorphisms in pfcrt, pfmdr1, dhfr genes and in vitro responses to antimalarials in Plasmodium falciparum isolates from Bangui, Central African Republic," American Journal of Tropical Medicine and Hygiene, vol. 75, no. 3, pp. 381-387, 2006.

[27] S. G. Staedke, H. Sendagire, S. Lamola, M. R. Kamya, G. Dorsey, and P. J. Rosenthal, "Relationship between age, molecular markers, and response to sulphadoxine-pyrimethamine treatment in Kampala, Uganda," Tropical Medicine and International Health, vol. 9, no. 5, pp. 624-629, 2004.

[28] K. Mugittu, S. Abdulla, N. Falk et al., "Efficacy of sulfadoxinepyrimethamine in Tanzania after two years as first-line drug for uncomplicated malaria: assessment protocol and implication for treatment policy strategies," Malaria Journal, vol. 4, Article ID 55, 2005.

[29] C. Goodman, S. P. Kachur, S. Abdulla et al., "Retail supply of malaria-related drugs in rural Tanzania: risks and opportunities," Tropical Medicine and International Health, vol. 9, no. 6, pp. 655-663, 2004.

[30] Ministry of Health and Social Welfare, "National Antimalarial guidelines for Tanzania,” 2006.

[31] G. M. Mubyazi, "The role of Research in changing the first-line antimalarial drug policy in Tanzania," Working Paper. Alliance for Health Policy and Systems Research (AHPSR), Geneva, 2003, http://www.alliancehpsr.int. 
[32] S. Abdulla, C. Goodman, P. Coleman, G. Mubyazi, N. Kikumbih, and T. Okorosobo, "The costs, effects and costeffectiveness of changing the first-line drug for the treatment of malaria in Tanzania," The Malaria Consortium, London School of Hygiene and Tropical Medicine/Liverpool School of Tropical Medicine, 2000. 


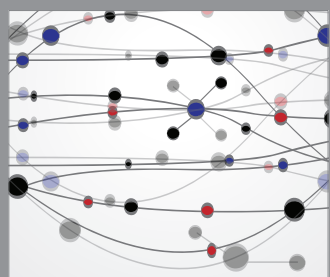

The Scientific World Journal
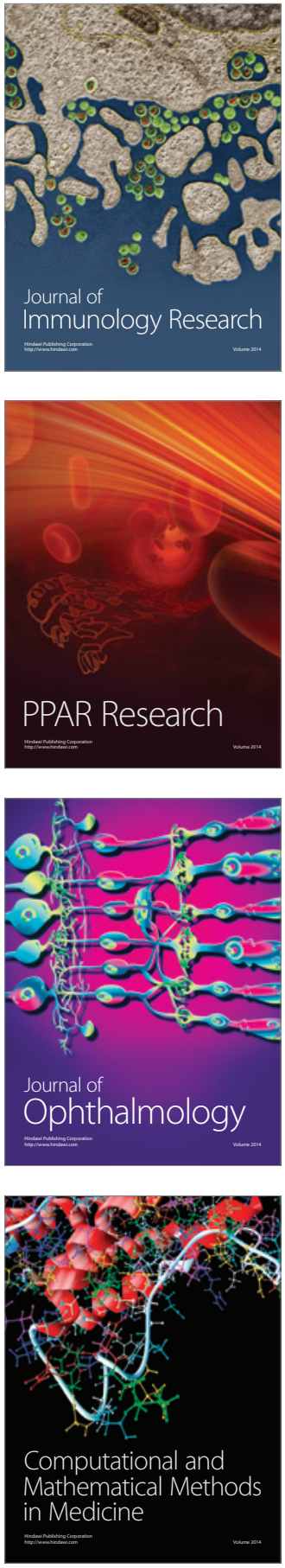

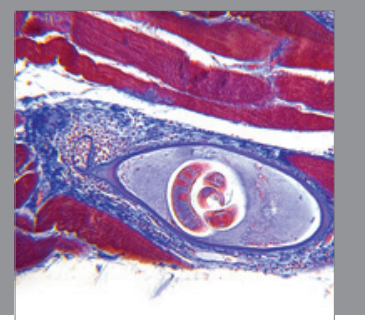

Gastroenterology

Research and Practice
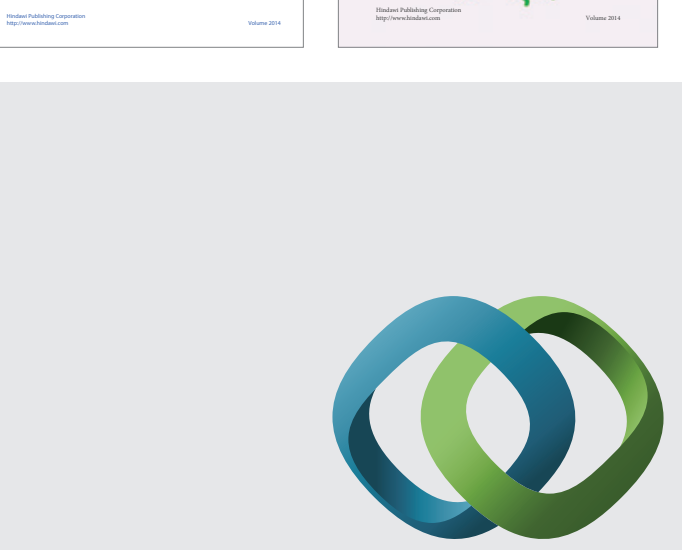

\section{Hindawi}

Submit your manuscripts at

http://www.hindawi.com
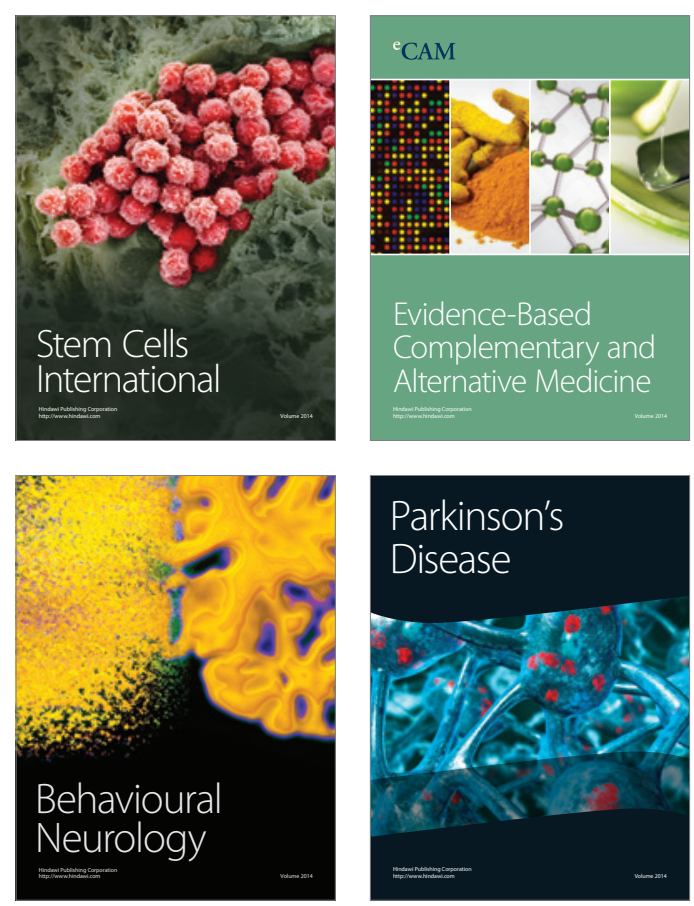

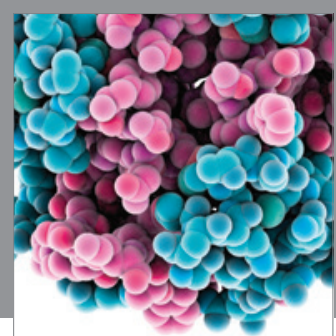

Journal of
Diabetes Research

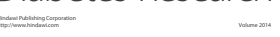

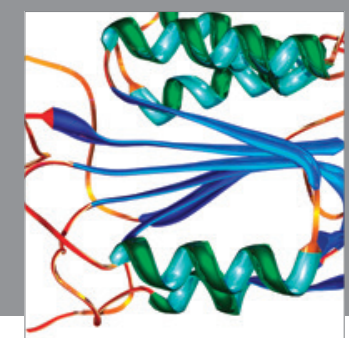

Disease Markers
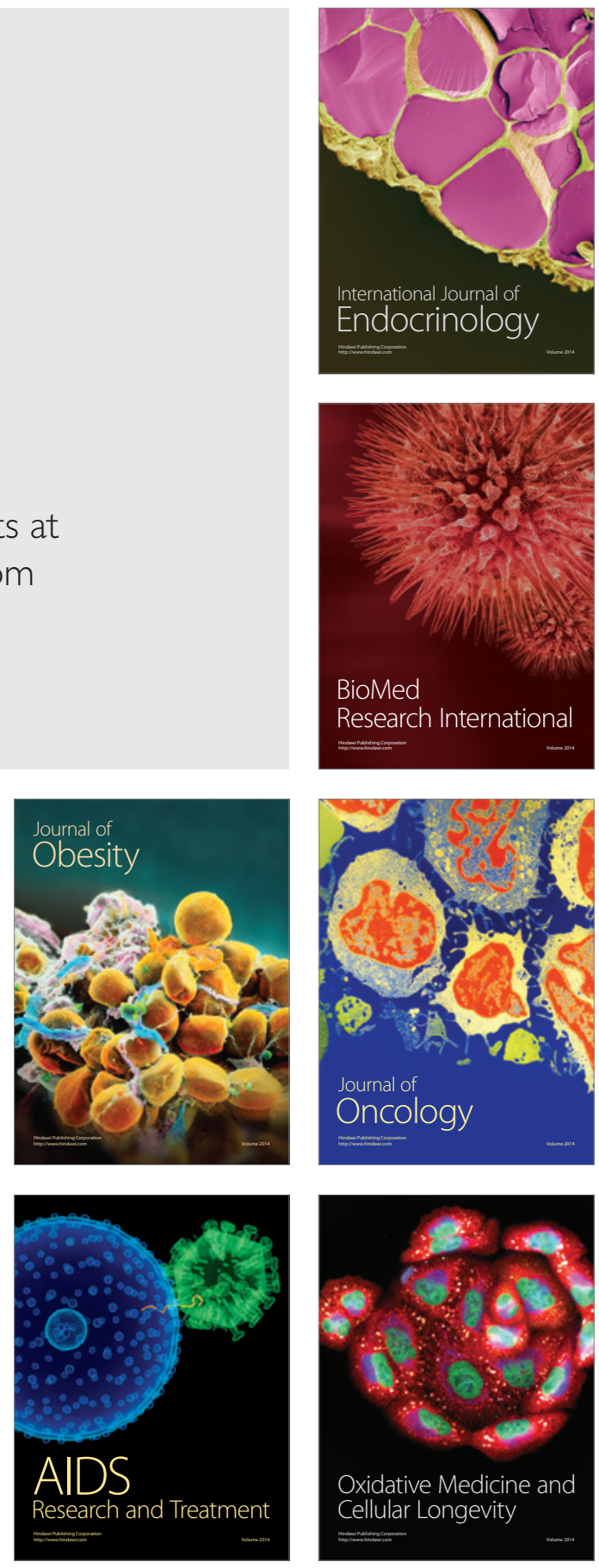